\title{
On the accuracy of PLIF measurements in slender plumes
}

\author{
Christina Vanderwel · Stavros Tavoularis
}

Received: 20 May 2014 / Accepted: 24 July 2014 - Author’s Accepted Version - doi: 10.1007/s00348-014-1801-x

\begin{abstract}
The purpose of this article is to assess the measurement uncertainty of the planar laser-induced fluorescence (PLIF) method and, as much as possible, to devise corrections for predictable biases. More specifically, we considered the measurement of concentration maps in cross-sections parallel to and normal to the axis of a slender plume containing Rhodamine $6 \mathrm{G}$ as a passive scalar tracer and transported by a turbulent shear flow. In addition to previously examined sources of error related to PLIF, we also investigated several unexplored ones. First, we demonstrated that errors would arise if the laser sheet thickness were comparable to or larger than the thickness of the instantaneous plume. We then investigated the effect of secondary fluorescence, which was attributed to absorption and re-emission of primary fluorescence by dye both within and outside the laser sheet. We found that, if uncorrected, this effect would contaminate the calibration as well as the instantaneous concentration measurements of the plume, and proposed methods for the correction of these errors and for identifying the instantaneous boundaries of the in-sheet dye regions.
\end{abstract}

\section{Introduction}

Planar laser-induced fluorescence (PLIF) is a popular method of studying the mixing and dispersion of passive scalars in turbulent flows. It consists of injecting an aqueous solution of fluorescent dye into water flow, illuminating a thin layer of the water with a laser sheet and recording its image, and constructing planar maps of dye concentration

C. Vanderwel $\cdot$ S. Tavoularis

Department of Mechanical Engineering, University of Ottawa,

Ottawa, Ontario, Canada

E-mail: stavros.tavoularis@uottawa.ca from the recorded maps of light intensity. PLIF is an attractive experimental technique, as it is non-intrusive, quantitative, and capable of providing two-dimensional scalar concentration maps. In addition, it can be fairly easily combined with other optical methods that can provide simultaneous measurements of flow velocity and temperature.

PLIF has been applied successfully by numerous previous investigators, some of which addressed various aspects of its uncertainty. For example, the uncertainty of PLIF image processing algorithms was discussed, among others, by Walker (1987), Ferrier et al (1993), Karasso and Mungal (1997), Cowen et al (2001), Melton and Lipp (2003), Webster et al (2003), Shan et al (2004), Crimaldi (2008), and Sarathi et al (2012). However, the requirements of uncertainty analysis in different studies have been quite diverse, depending on the objective and scope of the experiment, the flow conditions, the experimental configuration and other factors. In some studies, PLIF was used only qualitatively, for the purpose of detecting a scalar interface, without concern for quantifying concentration. In other studies, the experimental conditions were such that uncertainty that could be possibly introduced by some sources was essentially negligible. For these reasons, a general PLIF uncertainty analysis is still lacking, with several potential sources of uncertainty remaining unexplored.

Motivation for the work presented in this article arose during an experimental investigation of the diffusion of a slender passive scalar plume in turbulent flow, for which dye concentration measurements were collected on planes both parallel with and normal to the flow direction. The experimental setup and PLIF methodology are described in section 2, whereas some results have been presented in other articles (Vanderwel and Tavoularis, 2013, 2014a). In the course of these experiments, we first evaluated the 
concentration uncertainty introduced by sources discussed in the literature, but we also identified additional uncertainty sources and effects that have not been previously discussed, although intrinsic to the PLIF methodology.

The following section provides a brief description of the experimental facility, the properties of the dye used, and its illumination and recording methodology. In section 3, we present an idealized method for concentration measurement, which neglects the effects of concentration non-uniformity, light attenuation and secondary fluorescence. We then discuss each of these effects in turn in sections 4-6, providing limits of concentration non-uniformity level to avoid substantial errors and correction procedures for the effects of light attenuation and secondary fluorescence. In section 7, we present a method for identifying and discarding out-of-sheet dye, whereas, in section 8, we describe our method for concentration measurement that takes into consideration several corrections. Lastly, in section 9, we present a test of the accuracy of the PLIF measurements in a slender turbulent plume.

\section{The experimental facility, the dye and the PLIF instrumentation}

\subsection{The flow field}

The experiments were conducted in a recirculating water tunnel, having a test section with a free-surface, a width of $0.53 \mathrm{~m}$, a length of about $4 \mathrm{~m}$, and filled to a height of $0.46 \mathrm{~m}$ from the bottom. Uniformly sheared flow (USF), with a linear mean velocity variation, approximately homogeneous turbulence on a transverse plane, and Reynolds stresses that grew exponentially in the streamwise direction, was generated in the test section with the use of a shear generator. Details about the turbulent field have been presented previously by Vanderwel and Tavoularis (2011). Velocity measurements at the specific conditions of the present experiments were taken using stereoscopic particle image velocimetry (SPIV). A neutrally buoyant aqueous solution of fluorescent dye was injected isokinetically into the flow through a fine tube having an inner diameter of $1.83 \mathrm{~mm}$ and a wall thickness of $0.15 \mathrm{~mm}$, at a point approximately $2 \mathrm{~m}$ downstream of the shear generator, where the turbulence structure was fully developed (see figure 1). The undisturbed mean velocity at the point of dye injection was $U_{C}=$ $0.18 \mathrm{~m} / \mathrm{s}$, the mean shear was $d \bar{U}_{1} / d x_{2}=0.5 \mathrm{~s}^{-1}$, and the turbulent Reynolds number was $R e_{\lambda} \approx 150$. The integral lengthscale of the turbulence was comparable to the spacing of the shear generating apparatus, which was $L=25.4 \mathrm{~mm}$. (a) streamwise plane measurement

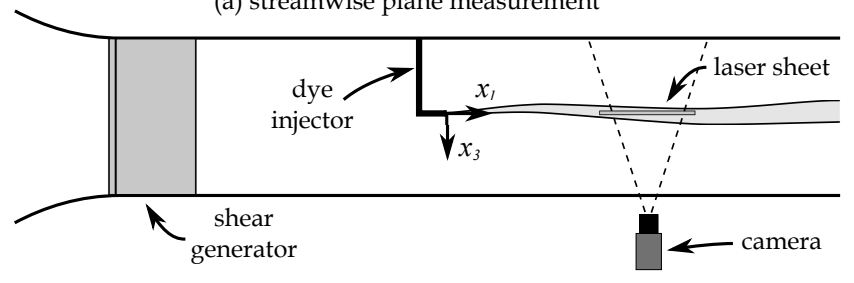

(b) normal plane measurement

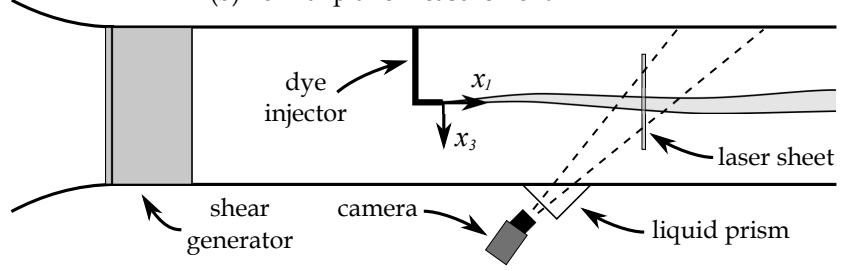

Fig. 1 Top view of the apparatus in the (a) streamwise and (b) normal plane configurations.

\subsection{The dye}

Rhodamine 6G (CAS: 989-38-8, A\&C American Chemicals Ltd., Montreal, Canada) was chosen as the dye tracer for these experiments. The absorption and emission spectra of Rhodamine 6G as measured by Würth et al (2012) for a concentration of approximately $0.4 \mathrm{mg} / \ell$ (which is comparable to concentrations measured currently in the plume) are plotted in figure 2. These spectra indicate an absorption peak at $525 \mathrm{~nm}$ and an emission peak at $554 \mathrm{~nm}$, in contrast to the values of $530 \mathrm{~nm}$ for the absorption peak and $560 \mathrm{~nm}$ for the emission peak as reported by Arcoumanis et al (1990); however, the shapes of the spectra are known to be sensitive to concentration and excitation wavelength, which could account for this discrepancy. The molecular diffusivity of the dye is $D=(4.0 \pm 0.3) \times$ $10^{-10} \mathrm{~m}^{2} \mathrm{~s}^{-1}$ (Gendron et al, 2008). Because the dye solution used was very dilute, its kinematic viscosity would be essentially equal to that of water in room temperature, namely $v=(1 \pm 0.05) \times 10^{-6} \mathrm{~m}^{2} \mathrm{~s}^{-1}$. Then, the Schmidt number of the dye can be estimated as $S c=v / D=2500 \pm$ 300 , which is a very large number.

Several tests were performed to determine the characteristics of the fluorescent dye under the specific conditions of the present experiments. Some tests aimed at determining a suitable dye concentration at the plume source, which had to be sufficiently high for the plume to be resolved accurately and sufficiently low for light absorption along the line of sight to be negligible. Dye solutions were prepared by mixing Rhodamine $6 \mathrm{G}$ powder with distilled water. Dye concentrations prepared in the laboratory were deemed to be reproducible within a precision of $0.5 \%$. Figure 3 is a photograph of dye solutions with different concentrations, illuminated by ceiling-mounted fluorescent tubes. At low concentrations, which were comparable to those used in the present experiments, the mixture appeared clear in laboratory light- 


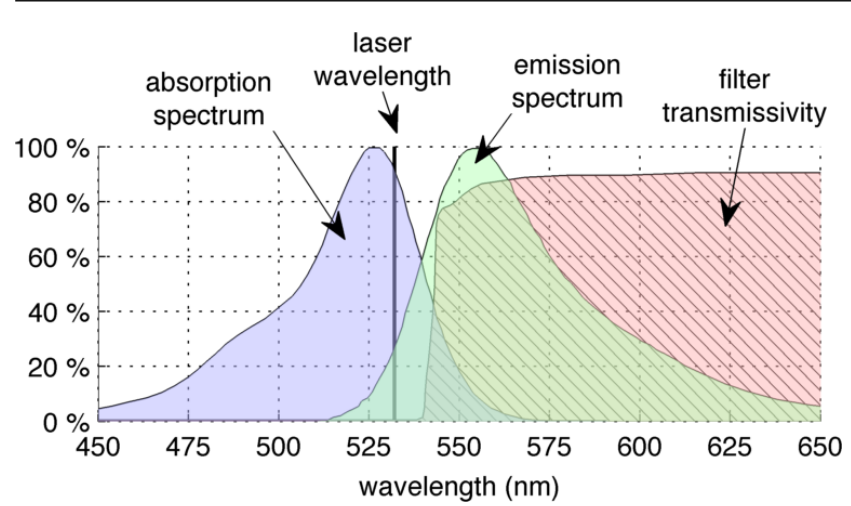

Fig. 2 The absorption and emission spectra of Rhodamine 6G (data from Würth et al, 2012, normalized by their maximum values) and the transmission spectrum of the optical filter (data from Lavision, Goettingen, Germany) with the wavelength of the Nd:YAG laser indicated by a solid black line.

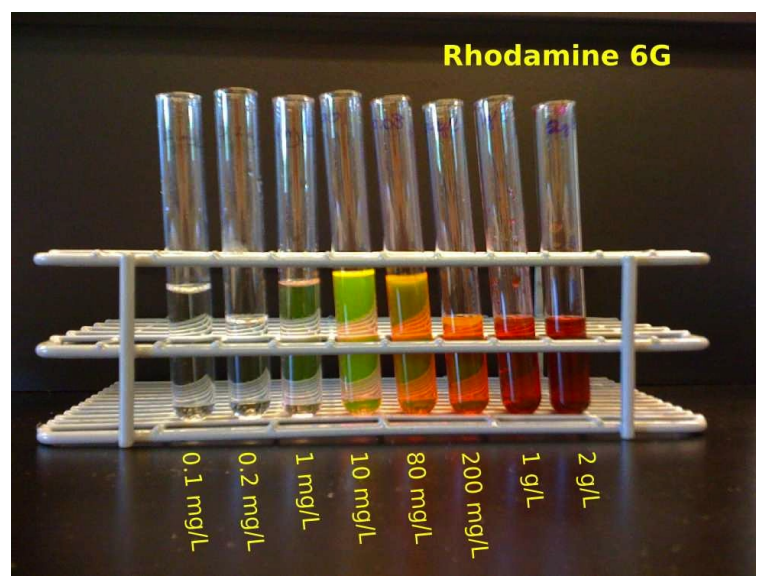

Fig. 3 Photograph of various concentrations of Rhodamine 6G mixed with water illuminated with standard ceiling-mounted fluorescent tube lights.

ing. Solutions with slightly higher concentrations produced some visible yellow-green fluorescence, as the room lighting emission spectrum overlaps with the dye's absorption spectrum. At higher concentrations, the fluorescence produced by the dye was quenched and the mixture had a red colour, like the Rhodamine $6 \mathrm{G}$ powder.

The following four minor effects, which could potentially affect the accuracy of concentration measurements, were examined in advance of the main tests.

a) Effect of water chlorination: To measure the rate of Rhodamine $6 \mathrm{G}$ bleaching by chlorine added to the water in the water tunnel (Webster et al, 2001), we prepared a mixture of dye with a concentration of $0.1 \mathrm{mg} / \ell$ using water from the water tunnel, which was equipped with an automated chlorination system that maintained the chlorine concentration at a nominal level of about $3 \mathrm{mg} / \ell$. The dye concentration of this mixture was measured until it decayed to half its initial value $C_{0}$. The measurements were fitted well by the exponential expression $C / C_{0}=e^{-t / t_{b}}$, where $t_{b}=3800 \mathrm{~s}$, corresponding to a half-life of $44 \mathrm{~min}$. During the convection time of the dye in the test section, its concentration would have decayed by about $0.3 \%$, which is much lower than the general concentration measurement uncertainty. In fact this chlorine bleaching effect is rather beneficial, as it reduces drastically the building-up of background concentration in the water tunnel.

b) Effect of $p H$ : Rhodamine $6 \mathrm{G}$ is known to be insensitive to $\mathrm{pH}$ in the range from 2 to 12 (Anaspec).

c) Effect of water temperature: According to the literature (Zhu and Mullins, 1992), the fluorescence of Rhodamine $6 \mathrm{G}$ is insensitive to temperature in the range below $120^{\circ} \mathrm{C}$, especially within the usual laboratory range.

d) Effect of photodecomposition: Unlike other fluorescent dyes, which are known to photobleach, Rhodamine $6 \mathrm{G}$ is insensitive to this effect (Arcoumanis et al, 1990; Crimaldi, 2008).

\subsection{Flow illumination and image recording}

The region of interest in the flow was illuminated by a Nd:YAG pulsed laser (Solo PIV 120XT, New Wave Research Inc., Fremont, CA, USA). The standard deviation of the pulse-to-pulse power fluctuations of the laser was found to be about 3\%; this value was determined by monitoring fluorescent emissions in a tank filled with a solution of dye with a uniform concentration over a $10 \mathrm{~min}$ interval and following a warm-up period of about $2 \mathrm{~min}$. Laser power fluctuations were deemed to have a much lesser effect on the time-averaged properties of the plume than other sources of error, to be discussed in section 9 .

Images of the flow were recorded using a digital sCMOS camera (pco.edge, PCO, Kelheim, Germany), which has an array of 2160 (height) $\times 2560$ diodes (i.e., approximately $5.5 \times 10^{6}$ pixels) and a 16 -bit pixel depth. The camera triggering was synchronized with the laser pulses. The laser was positioned underneath the water tunnel and the laser sheet was reflected by a mirror upwards through the glass wall. Measurements of the concentration were performed in cross-sections of the plume in planes parallel to the streamwise direction and normal to it, as illustrated in figure 1. The streamwise configuration measured the dye concentration in the $\left(x_{1}, x_{2}\right)$ plane at the centre of the tunnel, with the camera positioned normal to the measurement plane. Normal plane measurements in the $\left(x_{2}, x_{3}\right)$ plane were obtained by positioning the camera at an angle of $45^{\circ}$ to the laser sheet. A Scheimpflug adapter and a liquid prism were used to eliminate optical aberrations due to the oblique viewing angle (Prasad and Jensen, 1995).

The laser emission spectrum peaked at a nominal wavelength of $532 \mathrm{~nm}$, which is very close to the peak absorption wavelength of the dye. A long-pass optical filter 
with a cut-off wavelength of $540 \mathrm{~nm}$ (Part \# 1108573, LaVision, Goettingen, Germany) was placed in front of the camera lens to separate the fluorescence emitted by the dye from the laser light and light scattered by the SPIV seeding particles. Furthermore, the room lights were turned off during the experiment in order to eliminate the chance that any stray light would interfere with the measurements. The transmission spectrum of the optical filter is also indicated in figure 2; the filter was designed to block more than $99 \%$ of the laser light, while allowing approximately $75 \%$ of the fluorescent light to pass through to the camera.

\subsection{The calibration tank}

The camera output, pixel by pixel, was calibrated with the use of a small tank, having internal dimensions $307 \mathrm{~mm}$ (width) $\times 154 \mathrm{~mm}$ (thickness) $\times 210 \mathrm{~mm}$ (height), filled with a dye solution of uniform concentration $C_{\text {cal }}$, and submerged in the water tunnel in the field of view of the camera. Depending on whether the calibration was intended for use during streamwise or normal plane measurements, the thickness of the dyed fluid along the line of sight between the centre of the tank and the camera lens would be $77 \mathrm{~mm}$ or $109 \mathrm{~mm}$, respectively. In all cases, the distance over which the incident light travelled in the dyed fluid until it reached the centre of the tank would be $105 \mathrm{~mm}$.

\section{An idealized method for dye concentration measurement}

The measurement of dye concentration using the PLIF method is potentially subject to several bias and precision errors. As a starting point for evaluating the uncertainty of this method, we shall first present an idealized analysis, clearly identifying all underlying assumptions that are necessary for this analysis to be accurate.

Consider an elementary volume of fluid containing fluorescent dye with a uniform concentration $C$ and illuminated by laser light with a uniform radiant power flux $I$ (measured in $\mathrm{W} / \mathrm{m}^{2}$ ). Let the cross sectional area of this volume be $d A=d y_{1} d y_{2}$ and its thickness be $\mathrm{d} y_{3}$ (figure 4). The dye within the volume would emit "primary" fluorescent radiation that would be proportional to the product of the incident radiant power crossing the volume, the dye absorptivity $\varepsilon$ (measured in $\mathrm{m}^{-1}\left(\mathrm{~kg} / \mathrm{m}^{3}\right)^{-1}$ ) and the dye quantum efficiency $\phi$, i.e., the ratio of emitted to absorbed energy (Guilbault, 1990; Crimaldi, 2008). In the absence of light absorption along the line of sight (see section 5), the power flux of the radiation emitted by dye in this elementary volume and received by the camera lens, assumed to be far away from the illuminated region of the

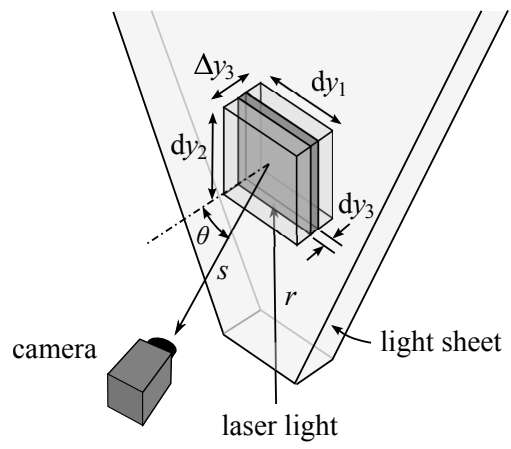

Fig. 4 Illustration of an elementary volume of fluid containing fluorescent dye and illuminated by a laser sheet.

flow, would be

$\mathrm{d} I_{f r}=h \phi \varepsilon I C \mathrm{~d} y_{3}$

where $h$ is a numerical coefficient that depends on the orientation of the camera. Now consider that illumination extends over a layer of fluid that has a thickness $\Delta y_{3}$. The radiant power flux of primary fluorescence that would be received by the camera lens would be

$I_{f r}=h \phi \varepsilon \int_{0}^{\Delta y_{3}} C\left(y_{3}\right) I\left(y_{3}\right) \mathrm{d} y_{3}$.

Assuming that the dye concentration were uniform over $\Delta y_{3}$, one may simplify the previous equation to

$I_{f r}=h \phi \varepsilon C \int_{0}^{\Delta y_{3}} I\left(y_{3}\right) \mathrm{d} y_{3}$.

Let us further assume that the only radiation received by the camera would be the one described previously. Each diode (i.e., a single pixel) of the camera provides as output a number $E$ between 0 and $2^{16}-1$ (i.e., 65535). Let $E_{0}$ be the output of the same diode in the absence of dye in the camera field of view; $E_{0}$ was typically about 100 for all diodes. The voltage difference $E-E_{0}$ would be proportional to the radiant power flux $I_{f r}$ received by that diode. This implies that the dye concentration at a point $\left(y_{1}, y_{2}\right)$ in the centreplane of the laser sheet would be related to the corresponding diode output as (Catrakis and Dimotakis, 1996; Webster et al, 2003)

$C\left(y_{1}, y_{2}\right)=\frac{\left[E\left(y_{1}, y_{2}\right)-E_{0}\left(y_{1}, y_{2}\right)\right]_{i}}{a_{i}\left(y_{1}, y_{2}\right)}$,

where $a_{i}$ is a pixel-specific calibration factor and the subscript $i$ denotes idealized conditions. Under the assumption that the idealized conditions are also met during calibration, the values of $a_{i}$ would be determined by calibration of the camera output in a tank having uniform concentration.

The previous analysis was based on several assumptions, whose violation may potentially influence significantly the accuracy of concentration measurements in slender 
plumes. For the evaluation of these assumptions, three particular effects need to be addressed: a) the effect of concentration non-uniformity across the illuminated fluid volume, b) the possible attenuation of incident radiation and fluorescence emitted by dye contained in the illuminated fluid volume, and c) the effect of radiation other than primary fluorescence, which would be added to the latter to produce the camera output. The importance of these effects during calibration and during measurement will be discussed in detail in following sections.

\section{Effect of concentration non-uniformity across the light sheet}

\subsection{Description of the effect}

Most analyses of PLIF measurements assume that the dye concentration changes sufficiently slowly for it to be constant across the light sheet produced by the laser. Although in a turbulent flow this condition can never be met exactly, in many cases it is satisfied approximately. In the present experiments, however, the instantaneous dye plume width was particularly small and the laser sheet thickness was not minimized for PLIF needs, but rather optimized for concurrent SPIV measurements. An example of an instantaneous concentration map measured in the streamwise plane is presented in figure 5. The instantaneous width of the plume was typically between 1 and $5 \mathrm{~mm}$, much smaller than the time-averaged plume width, but comparable to the laser sheet thickness, which was $2 \mathrm{~mm}$ (see the following subsection). Because calibration of the dye concentration measurement method was done using a tank filled with dye of uniform concentration, an error would occur when only part of the illuminated region during the experiment was occupied by the plume, or more generally when the concentration across the illuminated sheet was non-uniform.

\subsection{The laser sheet}

The variation of radiant power flux across the laser sheet was determined directly by illuminating a board painted with fluorescent paint and measuring the resulting fluorescence with the camera fitted with the optical filter. The fluorescent paint was a mixture of Rhodamine $6 \mathrm{G}$ powder, ethanol, and clear polyurethane paint, based on a recipe provided by LaVision (Goettingen, Germany). The radiant power flux variation was fitted well by the Gaussian function

$I\left(x_{3}\right)=I_{0} \exp \left[-\frac{x_{3}^{2}}{2 \sigma_{L}^{2}}\right]$,

where $I_{0}$ is the intensity on the centre of the sheet and the characteristic halfwidth of the sheet was $\sigma_{L} \approx 1 \mathrm{~mm}$.

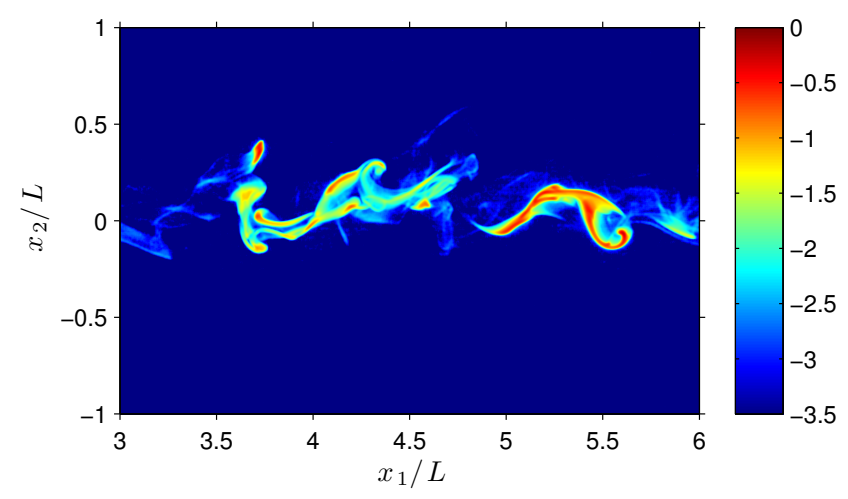

Fig. 5 Representative instantaneous map of dye concentration (in a logarithmic scale) with the optical setup in the streamwise plane configuration (figure 1a).

\subsection{Concentration error for a streamwise light sheet}

Let us consider a configuration in which the laser light sheet is parallel to a streamwise plane, as in figure 1a. For a simplified analysis, let us first assume that the plume has a Gaussian instantaneous concentration profile in the direction $x_{3}$ across the laser sheet, i.e.,

$C\left(x_{3}\right)=C_{p} \exp \left[-\frac{\left(x_{3}-g\right)^{2}}{2 \sigma_{P}^{2}}\right]$,

where $C_{p}$ is the peak concentration, $g$ is the position of the peak relative to the laser sheet centre and $\sigma_{P}$ is the instantaneous plume halfwidth (see figure 6). The radiant power flux emitted along a line of sight when the plume overlaps with the laser sheet can be calculated by (2). The voltage output of the camera would be

$E=k \int_{-\infty}^{\infty} I\left(x_{3}\right) C\left(x_{3}\right) \mathrm{d} x_{3}+E_{0}$,

where $k$ is a constant of proportionality. However, because calibration of the camera output was performed in a tank with uniform dye, this measurement would be interpreted as if the concentration were uniform across the laser sheet with an apparent value $C_{a}$, namely as

$E=k C_{a} \int_{-\infty}^{\infty} I\left(x_{3}\right) \mathrm{d} x_{3}+E_{0}$.

Equating the right-hand sides of (7) and (8), one may determine the apparent value of the concentration as

$C_{a}=\frac{\int_{-\infty}^{\infty} I\left(x_{3}\right) C\left(x_{3}\right) \mathrm{d} x_{3}}{\int_{-\infty}^{\infty} I\left(x_{3}\right) \mathrm{d} x_{3}}$,

which, in the case of Gaussian light intensity and concentration profiles, can be simplified to

$C_{a}=\frac{C_{p}}{\sqrt{1+\left(\sigma_{L} / \sigma_{P}\right)^{2}}} \exp \left[-\frac{\left(g / \sigma_{P}\right)^{2}}{2\left(1+\left(\sigma_{L} / \sigma_{P}\right)^{2}\right)}\right]$. 


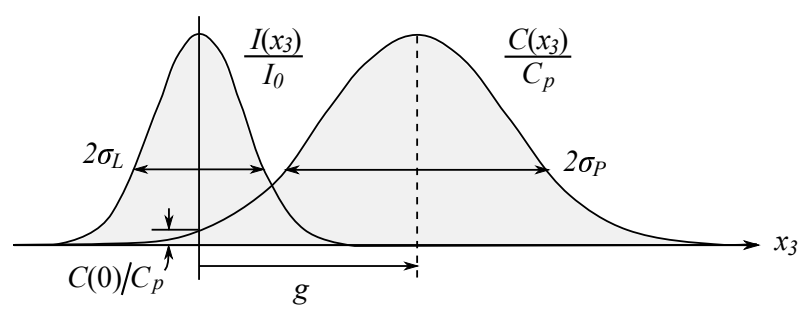

Fig. 6 Sketch of a plume of dye with a Gaussian concentration profile and a laser sheet with a Gaussian radiant power flux variation.

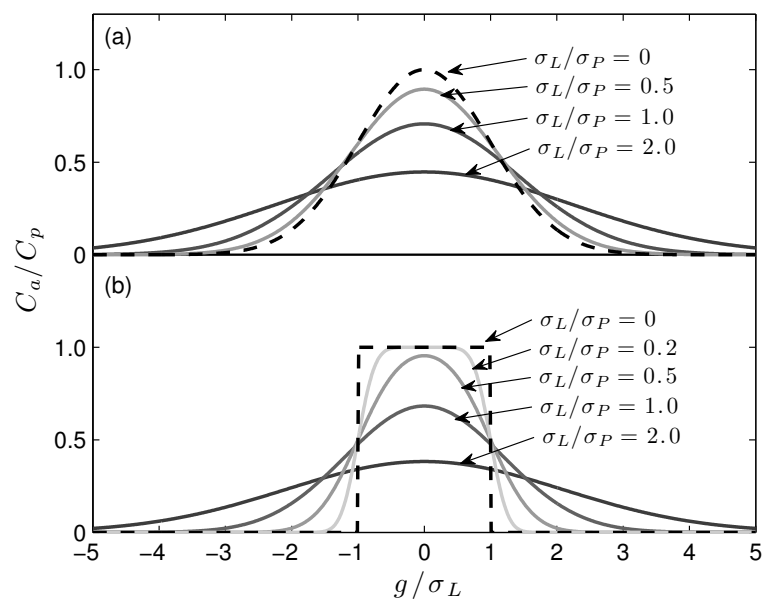

Fig. 7 The apparent concentrations of plumes with (a) Gaussian and (b) rectangular concentration profiles, having peak concentrations $C_{p}$ and widths $2 \sigma_{P}$ as functions of relative positions with respect to the laser sheet centreplane for different values of $\sigma_{L} / \sigma_{P}$.

The apparent concentration, normalized by the actual peak value, has been plotted in figure $7 \mathrm{a}$ as a function of plume position $g / \sigma_{P}$ for a few representative values of the ratio $\sigma_{L} / \sigma_{P}$. As $\sigma_{L} / \sigma_{P} \rightarrow 0, C_{a} \rightarrow C(0)$. It is important to note that the error $C(0)-C_{a}$ is positive when $g / \sigma_{P}$ is relatively small, but becomes negative for larger values of this ratio.

A similar analysis can be made for a plume that has a rectangular concentration profile with a value $C_{p}$ and a width $2 \sigma_{P}$. In this case, the apparent concentration would be

$C_{a}=\frac{C_{p}}{2}\left[\operatorname{erf}\left(\frac{\left(g / \sigma_{P}\right)+1}{\sqrt{2}\left(\sigma_{L} / \sigma_{P}\right)}\right)-\operatorname{erf}\left(\frac{\left(g / \sigma_{P}\right)-1}{\sqrt{2}\left(\sigma_{L} / \sigma_{P}\right)}\right)\right]$.

This relationship has been plotted in figure $7 \mathrm{~b}$ as a function of $g / \sigma_{P}$ for a few representative ratios of $\sigma_{L} / \sigma_{P}$. Again, as $\sigma_{L} / \sigma_{P} \rightarrow 1$, the apparent concentration becomes equal to the actual local concentration and the concentration error is positive when $g / \sigma_{P}$ is relatively small and negative for larger values of this ratio.

If the shape of the instantaneous plume concentration profile were known, as for the two cases considered previously, it would have been possible to devise corrections for this effect. However, because the actual plume profiles are very complex and unpredictable, this effect is nearly impossible to quantify or correct for. Finer dye filaments would be subject to a larger error than thicker dye filaments, and this error would manifest itself inhomogeneously and intermittently. The fact, however, that the local instantaneous error would sometimes be positive and sometimes negative would result in a partial cancellation of the overall error of the average local concentration. If the distance $g$ between the plume and laser sheet centreplanes were evenly distributed, the overestimated concentration at times when $g$ was relatively large would balance, partially or even totally (for the two cases of considered plume concentration profiles), the underestimated concentration when $g$ was relatively small and the ensemble-averaged concentration would end up having a lower or no error. In practice, however, the location of the plume centreplane would follow a quasi-Gaussian rather than uniform distribution, which would introduce a bias that would underestimate the average local concentration.

The matter is further complicated by other effects, which may introduce a bias in the concentration measurement. An additional error in plume concentration would be due to the depth of field of the camera, which would cause any fluorescence produced in front of or behind the centreline of the laser sheet to be blurred over neighbouring pixels. Whereas this effect would not introduce any error during calibration measurement in the tank of uniform concentration, it would likely cause an artefact that is equivalent to dye diffusion across the depth of field. All things considered, in the present experiment we would expect that, very near the source of the plume, where the plume was thin and did not meander significantly, the mean concentration would be underestimated, whereas further downstream, where the plume was much wider, errors would be less significant. This hypothesis will be verified in section 9 .

Although it may not be possible to devise accurate and general correction methods for the finite laser sheet thickness effect, it is possible to suggest a threshold for this thickness, relative to the plume width, below which the associated error may be neglected. Unlike the local concentration value, which may be either underestimated or overestimated, the apparent peak concentration $C_{a p}$ would always be lower than the actual peak concentration $C_{p}$. The ratios of these two values for Gaussian and rectangular plumes are, respectively,

$\frac{C_{a p}}{C_{p}}=\frac{1}{\sqrt{1+\left(\sigma_{L} / \sigma_{P}\right)^{2}}}$

and

$\frac{C_{a p}}{C_{p}}=\operatorname{erf}\left(\frac{\sigma_{P}}{\sqrt{2} \sigma_{L}}\right)$

These ratios have been plotted in figure 8 for a range of values of $\sigma_{L} / \sigma_{P}$. This figure shows that the error would be entirely negligible for $\sigma_{L} / \sigma_{P} \leq 0.3$ and would increase to val- 


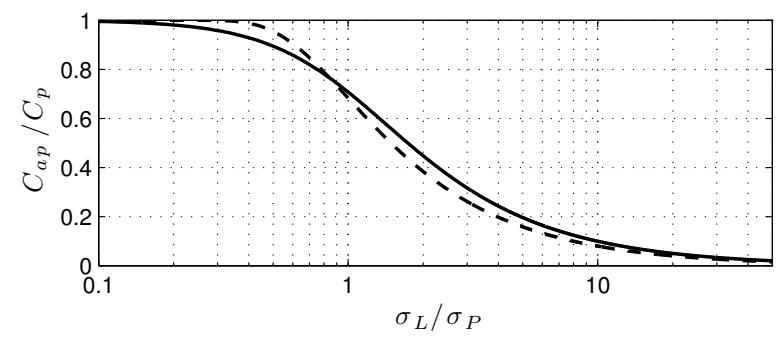

Fig. 8 Dependence of the ratio of apparent and actual peak concentrations of plumes with Gaussian (solid line) and rectangular (dashed line) concentration profiles on $\sigma_{L} / \sigma_{P}$.

ues comparable to $30 \%$ for $\sigma_{L} / \sigma_{P} \approx 1$. Taking into consideration that, for the present dye, which has a very low molecular diffusivity, the instantaneous plume concentration profile would more often be rectangular-like than Gaussian-like, we may assess that, when $\sigma_{L} / \sigma_{P} \leq 0.6$, the concentration error due to finite laser sheet thickness would not warrant specific correction and could be incorporated in the overall concentration measurement uncertainty.

\subsection{Concentration error for a normal light sheet}

In a normal-plane optical configuration, as in figure $1 \mathrm{~b}$, the thickness of the laser sheet would typically be much smaller than the streamwise extent of connected dye filaments. If the light sheet were viewed perpendicularly by the camera, one would then expect that the error due to dye non-uniformity across the laser sheet would be negligible. In the present setup, however, the camera viewing angle was oblique. With such a view, both the streamwise and the transverse intermittencies of the plume would introduce errors in the concentration measurement. Consequently, the error in this configuration could be, very roughly, comparable to the error in the streamwise viewing configuration. Measurements that will be presented in section 9 are compatible with this postulation.

\section{Effect of radiation attenuation}

The power flux of incident laser radiation transmitted through a fluid volume that contains an absorbing substance (dye) with a spatially varying concentration $C(r)$ along a path with a length $\Delta r$ would be attenuated according to the Beer-Lambert Law (Crimaldi, 2008)

$I_{a b}(r)=I \exp \left(-\varepsilon \int_{0}^{\Delta r} C(r) \mathrm{d} r\right)$,

where $I$ is the power flux of the incident light in the absence of attenuation. For a uniform concentration along the light

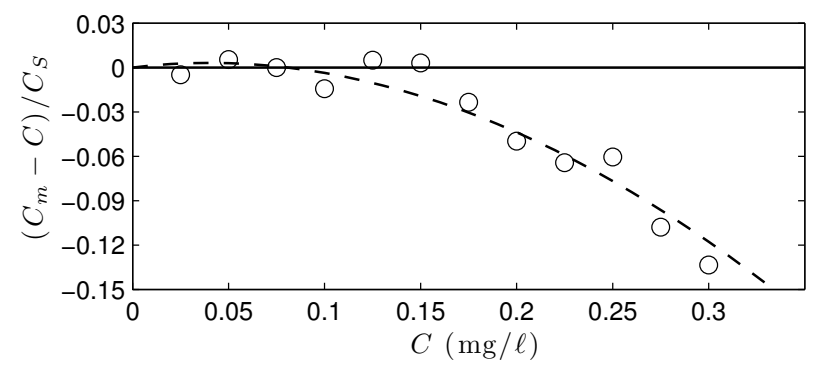

Fig. 9 Differences between the measured and actual concentrations in the calibration tank; the dashed line represents the best of (16) to the data; $C_{S}=0.3 \mathrm{mg} / \ell$.

path, this would become

$I_{a b}(r)=I \exp (-\varepsilon C \Delta r)$

Consequently, if one attempted to measure the local concentration $C$ while disregarding attenuation effects, one would get a lower value $C_{m}$ as

$C_{m}=C \exp (-\varepsilon C \Delta r)$.

The accuracy of this relationship was tested by taking concentration measurements in the calibration tank filled with solutions having uniform concentrations in the range from 0.025 to $0.3 \mathrm{mg} / \ell$ (Walker, 1987; Ferrier et al, 1993; Karasso and Mungal, 1997). The apparent concentration $C_{m}$ was calculated as the average of values from 150 images taken in the centre of the tank, where the length of the light path inside the tank was $\Delta r=105 \mathrm{~mm}$. The ratio $C_{m} / C$ is plotted vs. the actual concentration in figure 9 . The data could be fitted well (with differences having a standard deviation of $3 \%$ of the measured concentration) by (16) with $\varepsilon=4 \pm 1 \mathrm{~m}^{-1}(\mathrm{mg} / \ell)^{-1}$.

Based on the previous test, we chose the concentration of dye in the tank to be $C_{\text {cal }}=0.075 \mathrm{mg} / \ell$ during calibration of the camera; this value was deemed to be sufficiently large to produce a strong camera output but not large enough to result in significant radiation attenuation. The mean attenuation at the centre of the image with the tank filled with a dye solution having that concentration was approximately $(3 \pm 1) \%$, according to (16). The camera output during calibration was corrected for this attenuation of the incident laser light.

Equation (16) was also used to estimate the significance of light attenuation during the measurements in the plume. For the present work, the source concentration was chosen to be $C_{S}=0.3 \mathrm{mg} / \ell$. The maximum light attenuation, which would occur near the exit of the injection tube, was estimated to be $0.2 \%$, which is entirely negligible. Attenuation of the fluorescence emitted by the dye between the measurement plane and the camera lens is expected to have even less of an effect, considering that the absorptivity 
of the fluorescent signal is generally much lower than that at the wavelength of the laser (Lemoine et al, 1996).

\section{Effect of secondary fluorescence}

\subsection{Evidence for and possible sources of out-of-sheet fluorescence}

For the idealized analysis in section 3, it was assumed that the camera would receive only fluorescence radiation emitted by dye within the laser sheet. Nevertheless, dye outside the laser sheet was also recorded by the camera, as evident in the instantaneous image of the plume crosssection in the normal-plane configuration (figure 1b), shown in figure 14. Because of the oblique viewing angle, outof-sheet dye patterns are discernible to the left and right of the dye that would be illuminated by the laser sheet. Although out-of-sheet emissions were out of focus and at least an order of magnitude lower in magnitude than the peak emission of the in-sheet dye, they could be resolved fairly vividly by the camera, which had a very wide dynamic range, and were clearly visible when plotted with a logarithmic colour scale. If they remained unaccounted for, emissions from out-of-sheet dye would distort largely the shapes of the instantaneous and mean concentration maps of the plume and would bias the measurement of the total in-sheet dye mass.

The following possible causes for the visibility of outof-sheet dye were considered.

a) Ambient light: this was negligible, because the room lights were turned off during the experiments.

b) Laser light scattered by seed particles used for $S P I V$ : because of the very low number of particles in the illuminated plane, the scattered light was also considered to be negligible.

c) Laser light reflected from the free surface or the glass walls: this was not deemed to be significant, as it would remain largely within the measurement plane.

d) Primary fluorescent emissions by dye illuminated by the laser sheet: a small part of these emissions would be re-absorbed by the out-of-sheet dye, causing it also to fluoresce, albeit weakly. This secondary absorption and emission is made possible by the partial overlap of the absorption and emission spectra of the dye, a property which is true for most of the common fluorescence dyes used for PLIF. We believe that this secondary emission is the main source of out-of-sheet emissions which contribute to the total fluorescent intensity that reaches the camera lens. Of course, secondary fluorescence would not be restricted to out-of-sheet dye, but it would also be produced by in-sheet dye.
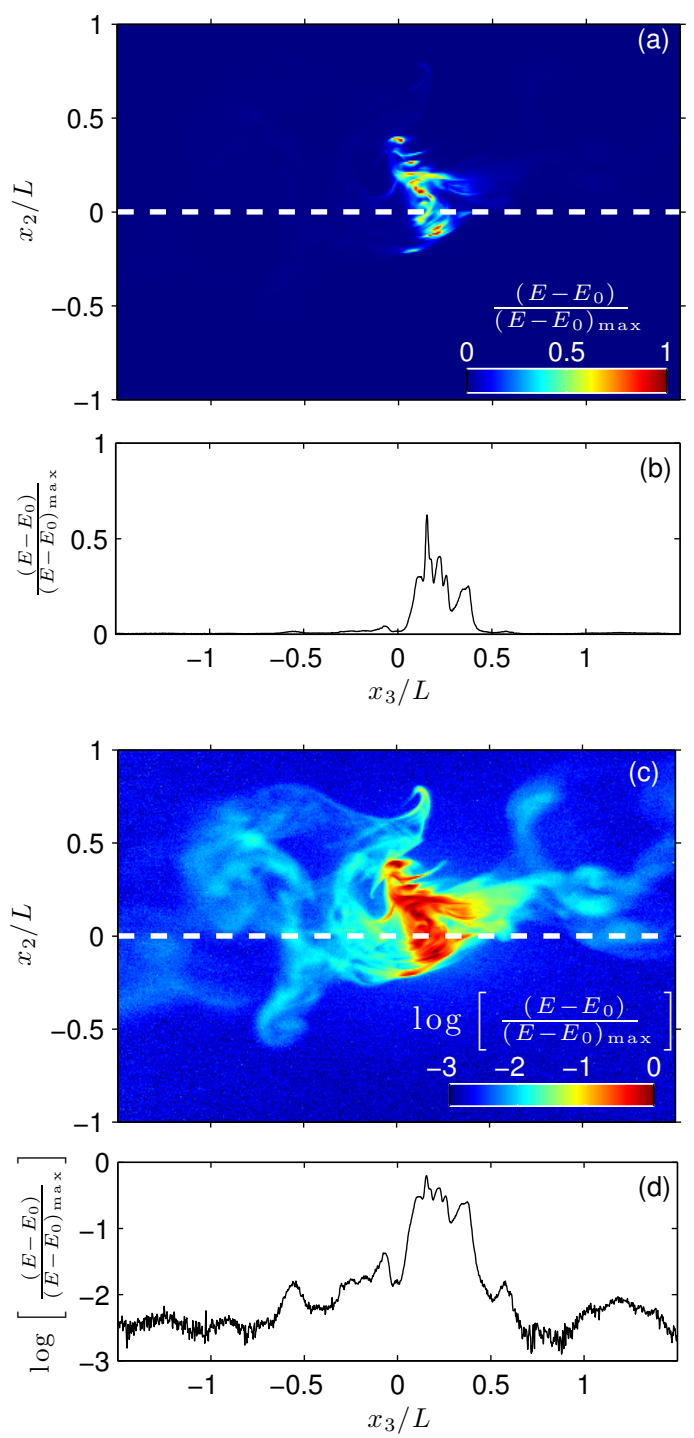

Fig. 10 Representative instantaneous maps of the net output $E-E_{0}$ of the camera viewing the plume in the normal plane configuration in (a) linear and (c) logarithmic scales. The values have been normalized by the maximum value $\left(E-E_{0}\right)_{\max }$ in the image. The dashed lines indicate the location of the profiles plotted in (b) and (d).

\subsection{Secondary fluorescence in the calibration tank}

The relative effect of secondary fluorescence upon the camera output was quantified directly by the following simple test. This test was performed in the calibration tank filled with a solution of Rhodamine $6 \mathrm{G}$ dye having a uniform concentration $C_{c a l}=0.075 \mathrm{mg} / \ell$. As shown in figure 11 , an opaque plate was mounted inside the tank parallel to the laser sheet so that it would block the view of part of the contained dye solution. By moving this plate in a direction normal to itself, it was possible to vary the thickness of the fluid layer that would be exposed to the camera, and hence the amount of out-of-sheet fluorescence. The length 
of incident laser light path in the tank remained the same for all positions of the plate and so did light attenuation.

For this test, we used a portable diode laser (S3 Krypton, Wicked Lasers) with the same emission wavelength $(532 \mathrm{~nm})$, laser sheet thickness $\left(\sigma_{L} \approx 1 \mathrm{~mm}\right)$, and laser sheet profile characteristics (see section 4.2) as the Nd:YAG laser used in the plume measurements.

The outputs of an array of $400 \times 100$ neighbouring pixels viewing the opaque plate were averaged to give the corresponding net output $E-E_{0}$. Although the diode laser had larger pulse-to-pulse power fluctuations than the Nd:YAG laser used for the plume measurements, in this test the measured values were corrected for possible temporal fluctuations in the laser intensity by comparison to a simultaneous reference measurement of the net output of a diode that viewed a layer of the fluid that was not blocked by the plate but had a uniform thickness equal to the total thickness of the fluid volume within the tank (see figure 11).

The total power flux received by the camera would be the sum of contributions of the primary fluorescence and the secondary fluorescence along the line of sight; the latter depends on the thicknesses of the two out-of-sheet dye layers in front of and behind the laser sheet, which may be made dimensionless by the laser sheet halfwidth $\sigma_{L}$. Therefore, one would anticipate that the net camera output would be a monotonically increasing function of the thickness of the exposed dye layer. Furthermore, one would also anticipate that the rate of increase of the net camera output would diminish with increasing thickness of the dyed fluid layer, as dye farther away from the laser sheet would emit less secondary fluorescence.

For the configuration of figure 11, the thickness of the dye layer in front of the laser sheet was fixed at $s_{1}=$ $2 \mathrm{~mm}$; this was the thinnest possible layer that would not clip the primary fluorescence emitted by dye within the laser sheet. Therefore, $E-E_{0}$ varied only with $s_{2}$, which took values between 1 and $127 \mathrm{~mm}$. Let $\left(E-E_{0}\right)_{p}$ be the hypothetical net output of the camera if there were only primary fluorescence. On condition of validation against the experimental results, one may assume that the power flux of secondary fluorescence varies as a power law of the emitting dye layer thickness. Then, for the configuration of figure 11, one could write the relationship

$$
E-E_{0}=\left(E-E_{0}\right)_{p}[1+\underbrace{b\left(\frac{s_{1}}{\sigma_{L}}\right)^{n}}_{\begin{array}{c}
\text { secondary } \\
\text { fluorescence } \\
\text { in front of sheet }
\end{array}}+\underbrace{b\left(\frac{s_{2}}{\sigma_{L}}\right)^{n}}_{\begin{array}{c}
\text { secondary } \\
\text { fluorescence } \\
\text { behind sheet }
\end{array}}] \text {, }
$$

where $b$ and $n$ are empirical constants to be evaluated by curve fitting to measurements.

Measurements of $E-E_{0}$ as a function of $s_{2} / \sigma_{L}$ are presented in figure 12. These measurements were

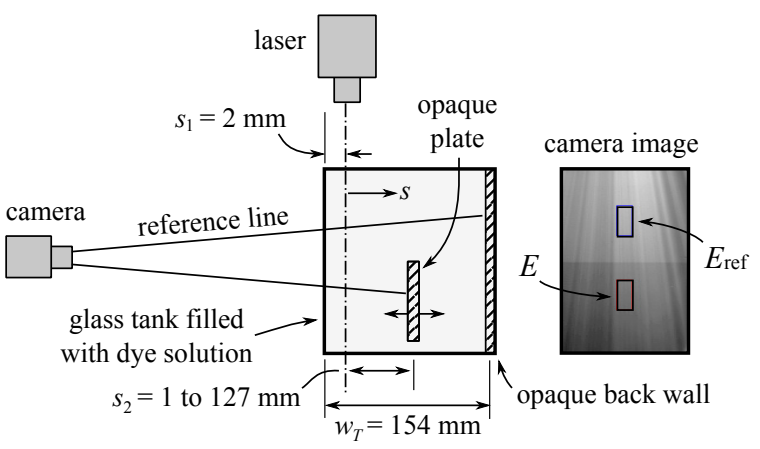

Fig. 11 Sketch of the experiment used to measure the effect of out-ofsheet fluorescence in a tank of uniform concentration fluorescent dye.

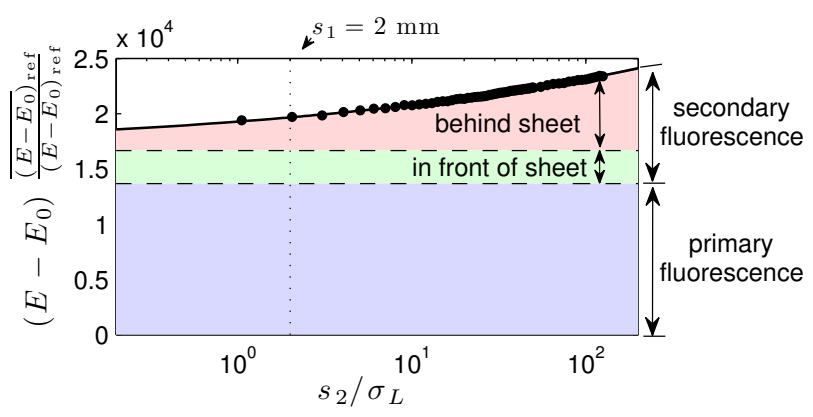

Fig. 12 The variation of the measured power flux $\left(E-E_{0}\right)$ of fluorescence measured in the test described in figure 11 as a function of the divider position $s_{2}$. The solid line represents the fit of (17) while shaded areas indicate the contributions of primary fluorescence and secondary fluorescence.

represented very well by the relationship

$E-E_{0}=c+b^{\prime}\left(\frac{s_{2}}{\sigma_{L}}\right)^{n}$

where $c=16684, b^{\prime}=2617$ and $n=0.20$. Then, the two remaining constants in (17) were determined as

$b=\left[c / b^{\prime}-\left(s_{1} / \sigma_{L}\right)^{n}\right]^{-1}=0.19$

and

$\left(E-E_{0}\right)_{p}=b^{\prime} / b=13678$.

\subsection{Camera calibration method}

Calibration was performed in situ for each pixel of the camera and took into consideration the effects of spatial variation in the laser sheet and optics, lens vignette, and any pixel-to-pixel offsets or gain variations in the camera. Separate calibrations were performed for the streamwise plane configuration and the normal plane configuration, as shown in figure 13. In both cases, the tank was filled with a solution of Rhodamine $6 \mathrm{G}$ dye having a uniform concentration $C_{c a l}=0.075 \mathrm{mg} / \ell$ and the laser sheet was 


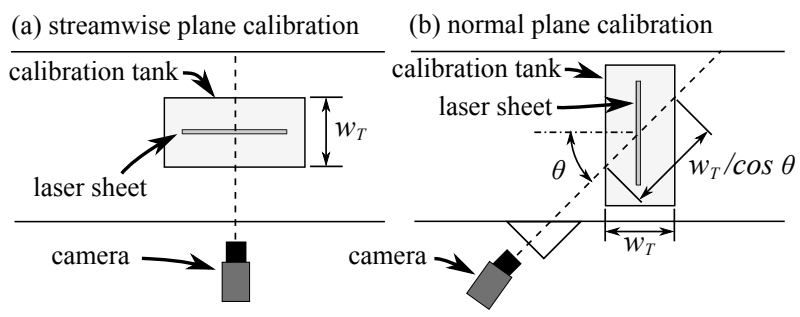

Fig. 13 Experimental configurations during calibration.

centred on the centreplane of the tank. The camera output during calibration, accounting for attenuation as explained in section 5, was a linear function of the concentration, having the same form as given by (4). The calibration factor of the camera could then be calculated from the camera output as

$a_{c a l}\left(y_{1}, y_{2}\right)=\frac{\left[E\left(y_{1}, y_{2}\right)-E_{0}\left(y_{1}, y_{2}\right)\right]_{c a l}}{C_{c a l}\left(y_{1}, y_{2}\right)}$.

This calibration factor would be specific to a particular calibration tank configuration and would introduce an error if used for the measurement of concentration in the plume. To render this calibration procedure suitable for general use, we will reference the camera response to idealized conditions, as described in section 3. Under such hypothetical conditions, the net camera output for a given pixel $\left(y_{1}, y_{2}\right)$ would be $\left[E-E_{0}\right]_{i}$ and its calibration factor would be $a_{i}$. These parameters would be related to the corresponding actual values as

$C_{c a l}=\frac{\left[E-E_{0}\right]_{i}}{a_{i}}=\frac{\left[E-E_{0}\right]_{c a l}}{a_{c a l}}$,

The idealized camera net output and the idealized camera calibration factors can be determined from the actual ones following corrections for absorption of the incident laser light by dye in the tank and for secondary fluorescence. Using the corresponding corrections that were derived previously, one gets for the streamwise plane calibration

$$
\begin{aligned}
\frac{a_{i}}{a_{c a l}} & =\frac{\left[E-E_{0}\right]_{i}}{\left[E-E_{0}\right]_{c a l}} \\
& =\left\{\exp \left(-\varepsilon C_{c a l} \Delta r\right)\left[1+2 b\left(\frac{w_{T}}{2 \sigma_{L}}\right)^{n}\right]\right\}^{-1},
\end{aligned}
$$

and for the normal plane calibration

$$
\begin{aligned}
\frac{a_{i}}{a_{c a l}} & =\frac{\left[E-E_{0}\right]_{i}}{\left[E-E_{0}\right]_{c a l}} \\
& =\left\{\exp \left(-\varepsilon C_{c a l} \Delta r\right)\left[1+2 b\left(\frac{w_{T}}{2 \cos \theta \sigma_{L}}\right)^{n}\right]\right\}^{-1}
\end{aligned}
$$

For the presently used calibration tank, the ratios of the idealized and actual camera calibration factors were 0.54 and 0.52 , respectively.

\section{Determination of the in-sheet plume boundaries}

As shown in figure 10, camera output maps displayed outof-sheet dye patterns that were undoubtedly produced by emissions of dye that were upstream and downstream of the in-sheet dye. These patterns would distort grossly the detected boundaries of the plume and would bias positively the local mass flux estimate. For this reason, we devised the following method to identify and remove out-of-sheet fluorescence.

Because the fluorescence from the out-of-sheet dye is non-uniform, an edge-detection-type algorithm would be unable to discriminate between in-sheet and out-of-sheet fluorescence. Instead, we used a thresholding technique, according to which the concentrations measured by all pixels in each instantaneous image that were lower than a specified threshold would be replaced by zero values. An example of a threshold chosen specifically to remove out-of-sheet dye emissions from the same representative instantaneous concentration map in the normal plane configuration as the one presented previously in figure 10 is shown in figure 14; the concentration contour at the value of this threshold, which has been superimposed on the concentration maps, illustrates that this approach resulted in a connected and plausible cross-section of the plume. Besides being subjective, user selection of an appropriate threshold for each image would be impractical, in view of the large number of images that were analysed. Instead, it would be more objective to determine an optimal threshold value for each ensemble of images with the use of an iterative algorithm. Another concern is that this approach would unavoidably exclude some low-concentration, insheet dye that, as a result of molecular diffusion, would be near the edge of the plume. To avoid significant overcompensation for this effect, one must choose a threshold that would, as much as possible, conserve the total in-sheet dye mass.

An algorithm to determine the optimal threshold for each set of images was developed, based on the histogram of the radiant intensity in the instantaneous images. This approach is similar to the one used by Prasad and Sreenivasan (1989) to determine the scalar interface of a turbulent jet. A representative histogram of an image in the present experiments is presented in logarithmic scales in figure 15a. This histogram had a peak at a concentration value of $0.25 \times 10^{-3} C_{S}$, which was the background noise level, and then extended towards higher concentration values up to a value slightly exceeding $0.1 C_{S}$. This particular plot does not provide a clear indication of the appropriate threshold value. In contrast, the frequency of occurrence of each concentration value, weighted by $C / C_{S}$ and plotted vs. $\log \left(C / C_{S}\right)$, allows one to determine the mass of dye within a concentration bandwidth by measuring the corresponding 

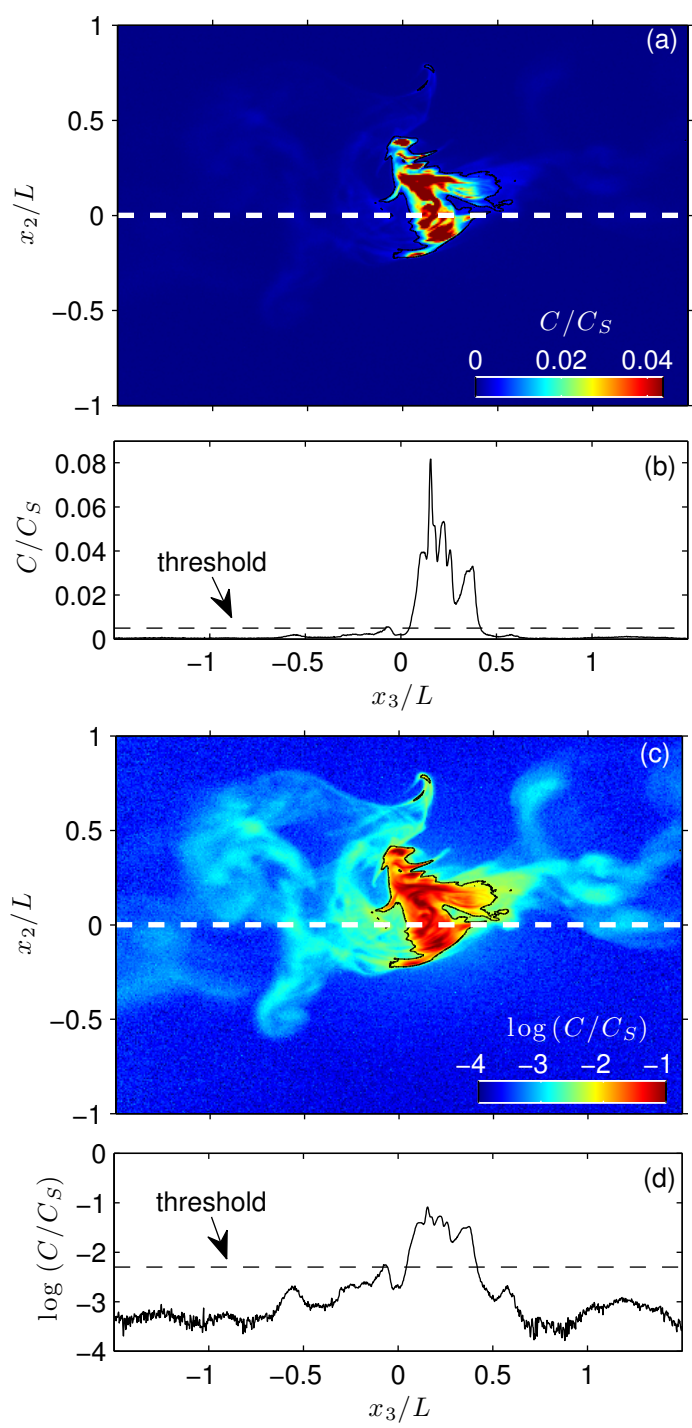

Fig. 14 Example of an instantaneous measurement of the plume showing maps of (a) $C / C_{S}$ and (c) $\log \left(C / C_{S}\right)$. The dashed line indicates the location of the cross-sections plotted in (b) and (d). A black line indicates the contour at a threshold of $\log \left(C / C_{S}\right)=-2.3$; this level is also indicated by a dotted line in the cross-section plots.

area under the curve. Such a plot (see figure 15b) clearly indicated the presence of three distinct peaks, which marked the background noise and the peak concentrations of the out-of-sheet dye and the in-sheet dye, respectively. The three peaks were separated by two local minima, which could serve for separating the three components of collected radiation intensity. To quantify objectively the equivalent dye masses that corresponded to each of these three components, we fitted the weighted histogram by the sum of three skew-normal functions (Azzalini, 1985) using a best fit algorithm in MATLAB. It is interesting to note that the contribution of background noise was entirely separated from the contribution of the in-plane dye emission, which confirms the camera manufacturer's specification of
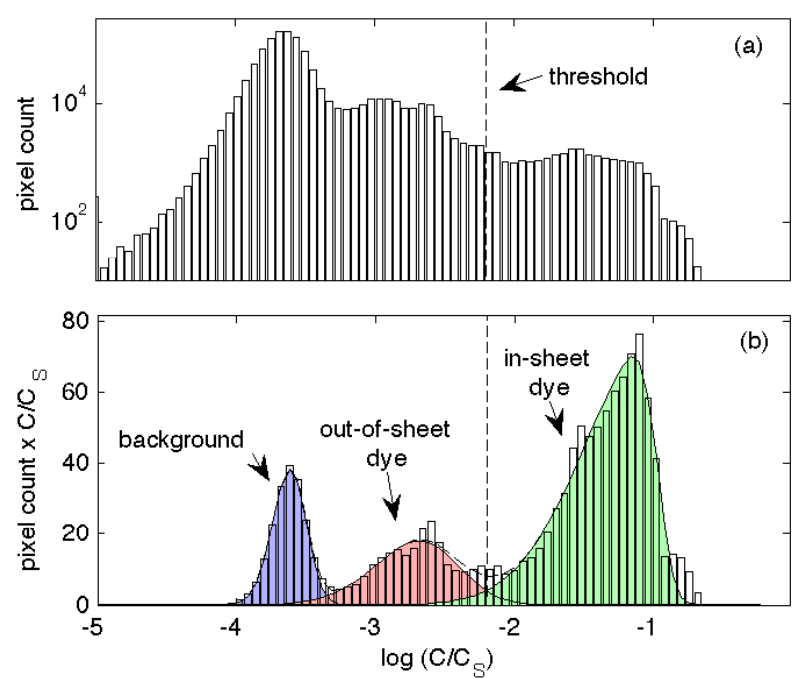

Fig. 15 (a) Histogram of the values in the instantaneous concentration map shown in figure $14 \mathrm{c}$ with bins uniformly spaced in the logarithmic scale and (b) the same histogram weighted by the values corresponding to the centre of each bin. The weighted histogram shows three distinct regions corresponding to the in-sheet dye, the out-of-sheet dye, and the background noise; skewed Gaussian functions have been fit illustrate the three regions. A carefully chosen threshold can be used to remove the majority of the out-of-sheet dye and background, while maintaining the majority of the signal from the in-sheet dye.

negligible noise. However, the concentration bandwidths for in-sheet emissions and out-of-sheet emissions overlapped, as expected. To separate the two contributions, we chose the threshold at a concentration value that was such that the area under the in-sheet curve below the threshold was equal to the area under the out-of-sheet curve that was above the threshold. This ensured that the mass of in-sheet dye would be conserved by the thresholding process. The computation of the optimal threshold for each instantaneous image was automated using a computer algorithm.

For the set of measurements presented in figures 14 and 15 , the optimal thresholds determined for each instantaneous image had an average of $\log \left(C / C_{S}\right)=-2.3$ and a standard deviation of 0.4 . Visual inspection of the resulting instantaneous in-sheet concentration maps confirmed that the plume appearance was realistic. Because the variability of the threshold for different images was relatively small, and also because some images did not contain any in-sheet dye at all, we reprocessed all images in each measurement set using the mean threshold value. This ensured that the average total mass of dye in the plume was not affected by the thresholding process, although the instantaneous mass could be somewhat distorted by the choice of suboptimal threshold; the standard deviation of this distortion was estimated to be approximately $10 \%$ of the mean total mass of the in-sheet dye.

It is worth noting that, as the plume spread downstream, the weighted histogram peak that corresponded to the 

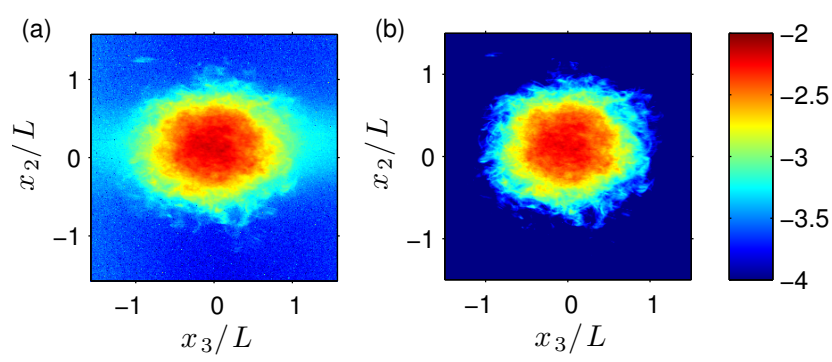

Fig. 16 The mean concentration map $\left(\log \left(\bar{C} / C_{S}\right)\right)$ from the same set of measurements as figure 14 determined (a) without and (b) with applying the thresholding correction.

background noise remained at essentially the same location, whereas both other peaks were shifted towards lower values. This increased the overlaps of the areas under the three skew-normal functions, which could potentially obscure the process of threshold identification. Nevertheless, this was not a problem for any of the measurements we investigated, because all weighted histograms were represented well by the sum of three distinct skew-normal functions and the algorithm for determining the threshold remained robust throughout all our experiments.

Representative maps of the mean concentration $\bar{C}$, determined without and with the thresholding algorithm are plotted in figure 16. The uncorrected map is much wider in the $x_{3}$ than in the $x_{2}$ direction, as a consequence of the fact that the vast majority of out-of-sheet fluorescence produced by the plume was viewed by the camera along the $x_{3}$ axis. The thresholding algorithm was effective in correcting this error and removed the halos from the flanks of the in-plane plume. It also removed the background noise. Removal of out-of-sheet fluorescence lowered the local mean concentration values by $5-8 \%$ of the peak $\bar{C}$, thus removing the corresponding positive bias.

Out-of-sheet fluorescence had an even lower effect on measurements of concentration fluctuations in the plume. The difference between the normalized standard deviations of the concentration $c^{\prime} / C_{S}$, determined without and with the thresholding correction, was less than $1 \%$ on average. The fact that the concentration fluctuation correction level is very small is a consequence of the fact that thresholding leaves unaffected large values of $C$, which are the main contributors to $c^{\prime}$.

Secondary fluorescence from out-of-sheet dye also contributed to the camera output in the streamwise plane configuration. This happened even when the plume was located completely outside of the laser sheet, due to meandering. In this configuration, the value of the optimal threshold cannot be determined by analysing the histograms of the instantaneous concentration measurements because the camera views of out-of-sheet dye and in-sheet dye were superimposed. To remove the contribution of out- of-sheet dye that would have biased positively the mean concentration measurements, we used the same thresholding algorithm with the same values of threshold as those determined from the normal plane measurements.

\section{Correction method for concentration measurements in the plume}

Concentration measurements in the plume would be subjected to the same sources of error as measurements in the calibration tank, as well as additional ones, which would be specific to each particular experimental configuration. The only way to apply the camera calibration procedure described in section 6.3 to measurements in the plume is by referencing all results to idealized conditions (section 3 ). The two main correctable errors that were identified during calibration were absorption of the incident laser light and secondary fluorescence. Because the present plume was very slender, absorption of the incident laser light across it would be negligible (see section 5). The effect of secondary fluorescence, however, was found to be significant and required correction.

The contribution of secondary fluorescence would depend on the instantaneous thickness of the plume, on the configuration of the plume measurement and on the location of the laser sheet with respect to the instantaneous plume axis. Close to the source, the plume thickness $w_{P}$ would be approximately equal to the injection tube inner diameter, which was roughly $2 \mathrm{~mm}$. Away from the source, $w_{P}$ would be smaller than the one obtained by time averaging of the plume position, which would be subjected to the effect of meandering. A better estimate of $w_{P}$ would be the relative plume width (Vanderwel and Tavoularis, 2014b), which was approximately $34 \mathrm{~mm}$ at the furthest downstream location of measurements $\left(x_{1} / L=35\right)$. Therefore, all along the present plume, its thickness was lower than the calibration tank width, which means that the effects of secondary fluorescence would be lesser during measurement than during calibration.

The two measurement configurations that were used in the present work are illustrated in figures $17 \mathrm{a}$ and $b$, respectively, for specific instances when the centre of the laser sheet would be on the plume axis. In the streamwise plane configuration, the ratio of the camera net output $E-E_{0}$ and its hypothetical value $\left[E-E_{0}\right]_{i}$ under idealized conditions, which is equal to the ratio $a / a_{i}$ of the corresponding calibration factors, would be

$$
\frac{a}{a_{i}}=\frac{\left[E-E_{0}\right]}{\left[E-E_{0}\right]_{i}}=\left[1+2 b\left(\frac{w_{P}}{2 \sigma_{L}}\right)^{n}\right] .
$$

In the normal plane configuration, $w_{P}$ would have to be replaced by $w_{P} / \sin \theta$. If, however, instead of being on the 


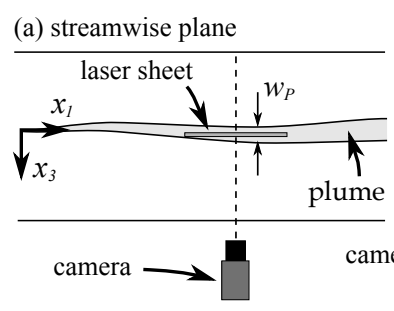

(b) normal plane with oblique view

(c) normal plane with end view

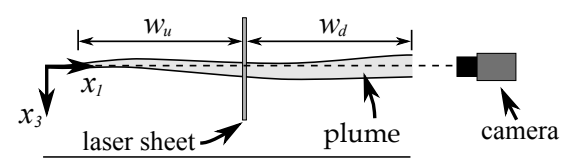

Fig. 17 Experimental configurations during measurements in the plume.

plume axis, the centre of the laser sheet were located on the edge of the plume, the calibration factor ratio would be

$\frac{a}{a_{i}}=\frac{\left[E-E_{0}\right]}{\left[E-E_{0}\right]_{i}}=\left[1+b\left(\frac{w_{P}}{\sigma_{L}}\right)^{n}\right]$

for the streamwise plane configuration and with $w_{P}$ replaced by $w_{P} / \sin \theta$ for the normal plane configuration.

The previous discussion demonstrated that the calibration factor ratio during concentration measurements in the plume would vary from one instant to the next, as the plume position fluctuated while the laser sheet remained fixed. A maximum local value of this ratio would occur when the centre of the laser sheet occupied the centreplane of the plume, while a minimum value would occur when the centre of the laser sheet was at the edge of the plume; contributions from images in which the plume was completely outside the laser sheet were successfully removed by the thresholding algorithm described in section 7 and so are no longer a concern. Table 1 shows the minimum and maximum values of the ratio $a / a_{i}$ at different locations along the plume for both configurations. A comparison of each pair of values shows that they were not very different and so it seems appropriate to chose the average of values determined from (25) and (26) for this ratio at each streamwise location. This approximation introduced an uncertainty of less than $10 \%$ on this correction factor.

Following this analysis, it becomes evident that the dye concentration in the plume can be calculated fairly accurately from the corrected output of the camera as

$C=\frac{E-E_{0}}{a}=\frac{E-E_{0}}{a_{c a l}} \frac{a_{c a l}}{a}$.

where the calibration factor ratio in the tank was found in section 6.3 to be $a_{c a l} / a_{i}=0.54$ and 0.52 for the streamwise and normal plane configurations, respectively. Ratios of $a_{c a l} / a$ are also presented in table 1 . Were the calibration coefficients for both the calibration and the plume measurements not corrected by this procedure, the

\begin{tabular}{ccccc}
\hline Configuration & $x_{1} / L$ & $w_{p} / \sigma_{L}$ & $a / a_{i}$ & $a_{\text {cal }} / a$ \\
\hline (a) & 4 & 4 & $1.34(1.25,1.44)$ & 1.37 \\
& 8 & 7 & $1.38(1.28,1.49)$ & 1.33 \\
& 11 & 10 & $1.41(1.30,1.52)$ & 1.31 \\
& 14 & 13 & $1.43(1.32,1.55)$ & 1.29 \\
& 17 & 16 & $1.45(1.33,1.58)$ & 1.27 \\
(b) & 20 & 19 & $1.47(1.34,1.60)$ & 1.26 \\
& 5 & 5 & $1.39(1.28,1.49)$ & 1.38 \\
& 12 & 11 & $1.45(1.33,1.57)$ & 1.32 \\
& 20 & 18 & $1.50(1.36,1.63)$ & 1.28 \\
(c) & 28 & 27 & $1.54(1.39,1.69)$ & 1.24 \\
& 35 & 34 & $1.56(1.41,1.72)$ & 1.22 \\
& - & - & 2.74 & 0.68 \\
\hline
\end{tabular}

Table 1 Calibration factor ratios for three configurations in the plume experiments. Values of $a / a_{i}$ outside parentheses are averages, whereas those in parentheses are minimum and maximum values, respectively.

plume concentration measurements would have been biased by factors equal to $\left(a_{c a l} / a\right)^{-1}$, which was typically between 0.7 and 0.8 .

Besides the two previous configurations, we have also examined a third configuration that was actually possible in our facility although not used in the present measurements. In this case, the camera would view a plane normal to the flow from a window at the downstream end of the test section (figure 17c). In such case, out-of-sheet dye would be present along the line of sight of the camera for long distances $w_{u}$ upstream and $w_{d}$ downstream of the laser sheet. Assuming that $w_{u}=w_{d}=2 \mathrm{~m}$, one can estimate that the calibration factor ratio would be $a / a_{\text {cal }}=1.48$, which means that concentration would be severely underestimated if corrections were not applied.

\section{A test of the overall accuracy of the present concentration measurements}

The accuracy of the concentration measurements in the plume was tested by comparing the measured total dye mass flow rates $\dot{m}_{m}$ in normal and streamwise cross-sections of the plume with the mass flow rate $\dot{m}_{i}$ of dye released from the injection tube. The latter was estimated as

$\dot{m}_{i}=C_{S} Q$,

where $Q$ was the water injection volume flow rate, measured with a rotameter.

The average mass flow rate of dye on transverse planes was estimated by integrating the corresponding mean concentration maps as

$\dot{m}_{m}=U_{c} \int_{-\infty}^{\infty} \int_{-\infty}^{\infty} \bar{C} \mathrm{~d} x_{2} \mathrm{~d} x_{3}$

where $U_{c}$ is the mean flow velocity in the centre of the channel. 


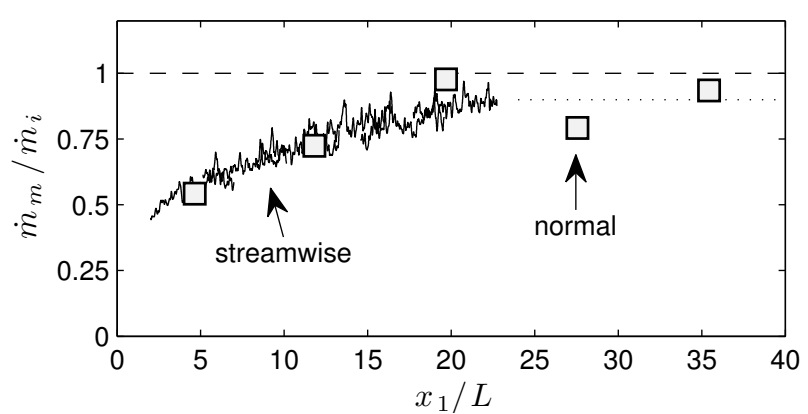

Fig. 18 Evolution of the measured dye mass flow rate, normalized by its value at the source; the solid line indicates measurements in a streamwise plane, whereas squares indicate measurements in crossplanes; the dotted line indicates the average of the data for $x_{1} / L \geq 20$, which was $\dot{m}_{m} / \dot{m}_{i} \approx 0.90$.

The average mass flow rate of dye on streamwise planes was calculated from profiles of $\bar{C}$ in the $x_{2}$ direction, under the assumption that the two-dimensional $\bar{C}$ map in the $x_{2}-x_{3}$ plane could be described by a Gaussian function as

$\bar{C}\left(x_{2}, x_{3}\right) \propto \exp \left[-\frac{x_{2}^{2}}{2 \sigma_{2}^{2}}-\frac{x_{3}^{2}}{2 \sigma_{3}^{2}}\right] ;$

the ratio of the plume half-widths was determined from the normal plane measurements as $\sigma_{3} / \sigma_{2}=1.25$ (Vanderwel and Tavoularis, 2014b).

The total masses of the mean concentration maps obtained on streamwise planes in the range $2<x_{1} / L<$ 23 as well as on normal planes at locations $x_{1} / L=5$, 12, 20, 28 and 35 are shown in figure 18. Both the streamwise and normal plane measurements were corrected for the effect of out-of-sheet fluorescence, as described in section 7. An observation that supports the consistency of the present analysis is that the corrected measurements in the two configurations essentially coincided, whereas there were significant differences between the corresponding uncorrected measurements.

Figure 18 clearly illustrates the fact that, near the plume source $\left(x_{1} / L=5\right)$, the measured mass flow rate, estimated from both streamwise and normal plane measurements, was significantly lower than the injected mass flow rate. This discrepancy is attributed to the effect of the dye concentration non-uniformity across the laser light sheet, as described in section 4, which was not accounted for in these estimates. This explanation is supported by the following arguments. Very close to the source, the instantaneous plume cross section would have a nearly rectangular concentration profile with a characteristic width equal to the inner radius of the injection tube, i.e. $\sigma_{P} \approx 0.92 \mathrm{~mm}$. Then, $\sigma_{L} / \sigma_{P} \approx 1.1$ and, according to figure 8 , the measured peak concentration would be approximately $63 \%$ of the true value $C_{S}$. This estimate of bias is consistent with the measured level of underestimation of mass flow rate at $x_{1} / L=5$ in figure 18 .
Away from the source $\left(x_{1} / L \gtrsim 20\right)$, the instantaneous plume filaments had shapes closer to a Gaussian than a rectangular one and had halfwidths in the range of $2 \mathrm{~mm} \lesssim \sigma_{P} \lesssim 5 \mathrm{~mm}$. This corresponds to $0.2 \lesssim\left(\sigma_{L} / \sigma_{P}\right) \lesssim 0.5$, so in this region the peak concentration would be equal to $89 \%-98 \%$ of the true value. This statement is compatible with the observation in figure 18 that the error in the measured mass flow rate due to concentration non-uniformity across the light sheet became small for $x_{1} / L \gtrsim 20$.

Although there was visible precision uncertainty (scatter) in the measurements for $x_{1} / L \gtrsim 20$, these measurements fluctuated around an average $\dot{m}_{m} / \dot{m}_{i} \approx 0.90$, which may be treated as a far-field asymptotic value. The remaining $10 \%$ discrepancy between the far-field measured dye mass flow rate and the mass flow rate at the source may be attributed to the approximations used in deriving corrections as well the anticipated combined uncertainty of unaccounted for aspects of the PLIF method; the latter include, among others, the uncertainties of a) injected dye concentration, b) local velocity used for mass flow rate calculation, c) laser power, which fluctuated with a standard deviation of $3 \%$, and d) the factor $a_{c a l} / a$, which depended strongly on the local plume width and could vary by $10 \%$. Although not perfect, the proposed methods of correction for secondary fluorescence and for out-of-sheet dye successfully drastically reduced any significant bias in the measured mass flow rate due to these effects.

In summary, one can draw the following conclusions concerning the accuracy of the present concentration measurements. All measurements have been corrected for errors due to out-of-sheet fluorescence, which is no longer a concern. They are deemed to be sufficiently accurate for $x_{1} / L \gtrsim 20$, where they are not subjected to significant error due to concentration non-uniformity across the laser sheet, but, for $x_{1} / L \lesssim 20$, the measurements would generally underestimate the true concentration due to this error, which increases with decreasing distance from the source.

\section{Conclusions}

In the previous sections, we have presented a detailed uncertainty analysis of PLIF measurements for the particular case of a slender plume in turbulent flow. In this analysis, we considered measurement planes both parallel and normal to the flow direction. In addition to uncertainties associated with the dye properties and dye attenuation, we analysed the error that would arise when the plume width is not much larger than the laser sheet thickness and the error that would be introduced by secondary fluorescence and by outof-sheet dye. None of these error sources has been addressed in previous literature. We found that the error associated with a relatively large laser sheet thickness can be very significant when the plume examined is very slender and 
suggested that, as a rule of thumb, the laser sheet thickness should be at most $1 / 3$ of the thickness of the thinnest filaments of dye in the plume. We found that this error would contaminate measurements in both the normal and the streamwise configurations, introducing a negative bias, which would increase with proximity to the plume source.

We also analysed the effect of secondary fluorescence, which we attributed to absorption and re-emission of primary fluorescence. We found that this effect influenced the concentration measurement by biasing the calibration measurements and proposed a method for correcting the calibration procedure, which if uncorrected would have resulted in a bias of $20-30 \%$. Furthermore, we found that secondary fluorescence emitted by out-of-sheet dye contaminated measurements of instantaneous concentration maps in the plume and we also proposed a correction method, which removed a bias of $5-8 \%$ in the mean concentration measurement. As an overall test of accuracy, we demonstrated that the corrected measurements conserved approximately the dye mass flow rate along the plume in the far field.

Acknowledgements Financial support by the Natural Sciences and Engineering Research Council of Canada is gratefully acknowledged.

\section{References}

Anaspec (Fremont, CA, USA) (2013) Rhodamine 6G Fluorescence Reference Standard [Online]. Available from: http://www.anaspec.com/products/product.asp?id=28765 [Accessed Sept 2013]

Arcoumanis C, McGuirk J, Palma J (1990) On the use of fluorescent dyes for concentration measurements in water flows. Exp Fluids 10:177-180

Azzalini A (1985) A class of distributions which includes the normal ones. Scand J Statist 12(2):171-178

Catrakis H, Dimotakis P (1996) Mixing in turbulent jets: scalar measures and isosurface geometry. J Fluid Mech 317:369-406

Cowen EA, Chang KA, Liao Q (2001) A single-camera coupled PTV-LIF technique. Exp Fluids 31:63-73

Crimaldi JP (2008) Planar laser induced fluorescence in aqueous flows. Exp Fluids 44:851-863

Ferrier AJ, Funk DR, Roberts PJW (1993) Application of optical techniques to the study of plumes in stratified fluids. Dynam Atmos Oceans 20:155-183

Gendron P, Avaltroni F, Wilkinson K (2008) Diffusion coefficients of several rhodamine derivatives as determined by pulsed field gradient-nuclear magnetic resonance and fluorescence correlation spectroscopy. J Fluoresc 18(6):1093-1101

Guilbault GG (ed) (1990) Practical Fluorescence, 2nd edn. Marcel Dekker, Inc.
Karasso P, Mungal M (1997) PLIF measurements in aqueous flows using the Nd:YAG laser. Exp Fluids 23(5):382-387

Lemoine F, Wolff M, Lebouche M (1996) Simultaneous concentration and velocity measurements using combined laser-induced fluorescence and laser doppler velocimetry. Exp Fluids 20(5):319-327

Melton L, Lipp C (2003) Criteria for quantitative PLIF experiments using high-power lasers. Exp in Fluids 35:310-316

Prasad A, Jensen K (1995) Scheimpflug stereocamera for particle image velocimetry in liquid flows. Appl Opt 34(30):7092-7099

Prasad RR, Sreenivasan KR (1989) Scalar interfaces in digital images of turbulent flows. Exp Fluids 7:259-264

Sarathi P, Gurka R, Kopp G, Sullivan P (2012) A calibration scheme for quantitative concentration measurements using simultaneous PIV and PLIF. Exp Fluids 52(1):247259

Shan JW, Lang DB, Dimotakis PE (2004) Scalar concentration measurements in liquid-phase flows with pulsed lasers. Exp Fluids 36(2):268-273

Vanderwel C, Tavoularis S (2011) Coherent structures in uniformly sheared turbulent flow. J Fluid Mech 689:434464

Vanderwel C, Tavoularis S (2013) Measurements of turbulent diffusion from a point source in uniformly sheared flow. In: Eighth International Symposium on Turbulence and Shear Flow Phenomena (TSFP8), Poitiers, France.

Vanderwel C, Tavoularis S (2014a) Measurements of turbulent diffusion in uniformly sheared flow. J Fluid Mech (submitted)

Vanderwel C, Tavoularis S (2014b) Relative dispersion of a passive scalar plume in turbulent shear flow. Physical Review E 89(4):041,005

Walker DA (1987) A fluorescence technique for measurement of concentration in mixing liquids. J Phys E: Sci Instrum 20:217-224

Webster D, Roberts P, Ra'ad L (2001) Simultaneous DPTV/PLIF measurements of a turbulent jet. Exp Fluids 30:65-72

Webster D, Rahman S, Dasi L (2003) Laser-induced fluorescence measurements of a turbulent plume. J Eng Mech-ASCE 129:1130-1137

Würth C, González MG, Niessner R, Panne U, Haisch C, Genger UR (2012) Determination of the absolute fluorescence quantum yield of Rhodamine $6 \mathrm{G}$ with optical and photoacoustic methods-providing the basis for fluorescence quantum yield standards. Talanta 90:3037

Zhu Y, Mullins O (1992) Temperature dependence of fluorescence of crude oils and related compounds. Energ Fuels 6(5):545-552 